http://dx.doi.org/10.4314/ajid.v7i1.3

\title{
EOSINOPHILIC MENINGITIS IN A 10-YEAR OLD NIGERIAN BOY: A CASE REPORT
}

\author{
B. Adeola Animasahun ${ }^{\mathrm{a}}$, Olugbenga H Gbelee ${ }^{\mathrm{a}}$, Adaobi Solarin ${ }^{\mathrm{b}}$, Ajibike OA Ikuerowo ${ }^{\mathrm{c}}$, Adeola M \\ Raji $^{\mathrm{d}}$, Peter Ubuane ${ }^{\mathrm{c}}$, EO Kingsley ${ }^{\mathrm{d}}$, Fidelis O Njokanma ${ }^{\mathrm{a}}$, \\ ${ }^{a}$ Department of Paediatrics and Child Health,Lagos State University College of Medicine. \\ Ikeja, Lagos, Nigeria, ${ }^{b}$ Department of Paediatrics and Child Health,Reddington Multispecialist \\ Hospital Ikeja, Lagos, Nigeria, ${ }^{\mathrm{c}}$ Department of Paediatrics, Lagos State University Teaching Hospital \\ Ikeja, Lagos, Nigeria, ${ }^{\mathrm{d}}$ Department of Microbiology and Parasitology, Lagos State University \\ College of Medicine Ikeja, Lagos, Nigeria. \\ *E-mail: deoladebo@yahoo.com (BA Animasahun)
}

\begin{abstract}
Eosinophilic meningitis is rare, commonly caused by invasion of the central nervous system by helminthes. The present case is that of a 10-year-old boy who presented with history of generalized pruritus not associated with skin eruptions, followed by pain and weakness of the extremities and loss of consciousness. Patient did not receive BCG vaccination. Initial clinical evaluation was suggestive of tuberculous meningitis but cerebrospinal fluid analysis revealed eosinophilic meningitis. Patient made remarkable improvement with treatment with no sequel.
\end{abstract}

Key-words: Eosinophilic Meningitis, Tuberculous Meningitis, central nervous system,

\section{Introduction}

Eosinophilic meningitis is defined by the presence of 10 or more eosinophils/ $\mu \mathrm{L}$ in the CSF or eosinophilia of at least $10 \%$ of the total CSF leukocyte count (Lo and Gluckman, 2003)(Liu et al, 2011). Invasion of the central nervous system by helminthic parasites is the most common cause, but other infections as well as several non-infectious aetiologies have also been associated. It has been reported in patients with neurosyphilis and tuberculous meningitis although reports of these are rare (Lo and Gluckman, 2003)(Liu et al, 2011).

The authors are not aware of any case previously reported in a Nigerian child and hence report the case of a ten year old boy for its rarity and interest.

\section{Case report}

The patient is a 10 year- old boy, the youngest of three siblings, who presented with two weeks history of generalized pruritus not associated with skin eruptions, followed by pain and later weakness in both legs which further deteriorated to loss of ability to neither stand nor walk. This was followed by constipation with progressive abdominal distension, weight loss, low-grade fever, straining at micturition and urinary retention. There was no history of chronic cough or contact with any adult with chronic cough, however, Patient did not receive BCG vaccination. Other siblings were well. The family does not eat raw meat of any kind but seafood was eaten occasionally.

Examination revealed a wasted, febrile $\left(37.8^{\circ} \mathrm{C}\right)$ child with signs of meningeal irritation. There was hypotonia and hyporeflexia in the lower limbs with power of Grade 2. Sensation was intact but he had left $6^{\text {th }}$ cranial nerve palsy. There was also moderately distended abdomen with generalized tenderness, tympanitic percussion notes and suprapubic fullness, reduced anal sphincter tone, smooth rectal mucosa with formed faeces in rectum.

Initial diagnostic considerations included transverse myelitis, Potts disease, poliomyelitis and Gullain Barre syndrome. Over the next three days, his clinical condition deteriorated; his speech became slurred and irrational, there was complete loss of power in all extremities and the patient had lapsed into unconsciousness. The patient was then commenced on treatment for Tuberculous meningitis (using rifampicin, isoniazid, pyrazinamide, pyridoxine and prednisolone), to rule out encephalitis, however cerebrospinal (CSF) analysis confirmed eosinophilic meningitis for which patient was treated. Mantoux test was negative, plain abdominal X-ray and Abdominal USS, skull X-ray and MRI of the Brain were normal, Stool and Urine microscopy were negative for ova and parasite. He made remarkable improvement with treatment, was discharged and is well with no sequel on follow up. 
http://dx.doi.org/10.4314/ajid.v7i1.3

\section{Discussion}

Eosinophilic meningitis is a rare condition. Although tissue-migrating helminthes are the commonest cause, eosinophilic meningitis has been reported in patients with neurosyphilis and Tuberculous meningitis although reports in these are rare. (Lo and Gluckman, 2003; Liu et al., 2011 ; Louisiana Dept of Health\& hospitals). It can also result from non-infectious origin such as drugs (ciprofloxacin, ibuprofen, intraventricular vancomycin, gentamicin, iophendylate dye used in myelography) and malignancies (Hodgkin lymphoma, Non-Hodgkin lymphoma, eosinophilic leukemia). Patients with ventriculo-peritoneal shunts can also develop CSF eosinophilia due to an allergic reaction to the shunt material (Louisiana Dept of Health \& Hospitals). In the current patient, it was difficult to pinpoint the specific cause, a probable cause was Angiostrongylus cantonensis, based on clinical signs and his eating habits. The principal etiologic agent of human cause of eosinophilic meningitis is Angiostrongylus cantonensis (Lo and Gluckman., 2003; Liu et al., 2011 ; Louisiana Dept of Health\& hospitals; Diao et al., 2009) a lung worm of rats, which was first detected in rats in Canton, China in 1933. The first human case was detected in Taiwan in 1944, first reported in the United States in 1985, with a probable introduction by infected rats from ships docking in New Orleans, Louisiana, during the mid-1980s. It is found in Southeast Asia, the South Pacific, Japan, Taiwan, Egypt, Ivory Coast, and Cuba. Infection is acquired by eating raw or undercooked freshwater snails, slugs, prawns, or crabs containing infectious 3rd-stage larvae (Louisiana Dept of Health \& hospitals).

Gnathostoma infections which is another cause of eosinophilic meningitis are found in Japan, China, India, Bangladesh, and Southeast Asia. It is acquired by eating undercooked or raw fish, frog, bird, or snake meat. Worm have been reported in rodents in various parts of the world, it was reported in a rodent in Port-harcourt Nigeria in 1989 (Louisiana Dept of Health \& hospitals). Adult worms reside and lay eggs in the pulmonary arteries of rats. First-stage larvae hatch and migrate via the trachea and gastrointestinal tract into the faeces. Snails and slugs that feed on rodent excrement serve as intermediate hosts and allow the larvae to moult into infective third-stage forms. Rats and humans become infected by consuming third stage infective larvae. Humans accidentally acquire infection by consuming raw tissues of infected molluscs: either by ingesting improperly cooked intermediate hosts (snails and slugs) or through ingestion of food (salad greens) contaminated by slug or snail slime or containing minute slugs or ingestion of raw paratenic hosts (freshwater shrimp, land crabs, frogs) that have eaten infected molluscs (Louisiana Dept of Health\& hospitals).

In humans, the third-stage larvae are transported via the bloodstream to the central nervous system where they burrow into the neural tissue, inciting an inflammatory response that eventually kills the parasites. When eosinophilic meningitis results from helminthic infestation, patients become ill $1-3 \mathrm{wk}$ after exposure, because the parasites migrate from the gastrointestinal tract to the CNS. Common concomitant findings include fever (low-grade or none at all), peripheral eosinophilia, nausea, vomiting, abdominal pain, creeping skin eruptions, or pleurisy. (Liu et al., 2011; Louisiana Dept of Health \& hospitals). Neurologic symptoms may include headache, meningismus and ataxia. Cranial nerves are rarely affected; particularly the optic and facial nerves. Severe headache is the main complaint. The headache is intermittent, intractable, bitemporal, or occipital and continues throughout the clinical illness. Paraparesis or incontinence can result from radiculitis or myelitis. Paresthesias of the trunk and extremities commonly manifest as exaggerated sensitivity to touch and may persist for several weeks or months. Altered consciousness, generalized weakness and flaccid paralysis are rare. The current patient presented with some of these features (low grade fever, headache, abdominal pain, he also had cranial nerve palsies, paraparesis followed by flaccid paralysis and then altered consciousness).

The clinical features of eosinophilic meningitis caused by helminthes can be divided into four stages: latent stage lasts 3 to 36 days (average 15 days) with no obvious clinical signs; Prodromal stage is mild, it may include fever, headache, dizziness, abdominal pain, diarrheoa and lack of strength. Symptoms can resolve without treatment; acute stage is characterized by severe fever and headache with nausea, vomiting and abnormal sensations of the skin (e.g., numbness, pain, tingling, burning). Stiff neck, numbness of face or limbs, photophobia and diplopia may occur. (Liu et al., 2011). This phase may last from one week to 2 months or even longer; in the recovery stage, symptoms start to resolve and patients gradually regain normal status. Recovery can last for several weeks, depending on the individual. Some measurements (such as eosinophils counts, CSF pressure, positive head MRI signals and lung shadow) and mildly abnormal sensations could last longer. (Liu et al., 2011). The standard treatment for eosinophilic meningitis caused by A. cantonensis infection has been controversial (Tsai HC et al.,2011). A. cantonensis is susceptible to broad-spectrum anthelminthics, e.g., thiabendazole, mebendazole, albendazole, and ivermectin (Louisiana Dept of Health\& hospitals; Diao Z et al., 2009). However, some think these drugs should not be used in acute stage because clinical deterioration or death can result from a reaction to dead or dying worms in the brain (Louisiana Dept of Health\& hospitals). Analgesics are administered for headache and radiculitis, and CSF removal or shunting are beneficial to relieve hydrocephalus, if present ( Liu et al., 2011; Louisiana Dept of Health\& hospitals). Steroids may decrease the duration of headaches in adults with eosinophilic meningitis (Prober, 2007), the current patient had dexamethasone and serial CSF removal but was not given antihelminthics during the acute illness.

Eosinophilic meningitis caused by helminthes has a good prognosis, $70 \%$ of patients improve sufficiently to leave the hospital in 1-2 weeks. Mortality associated with eosinophilic meningitis $<1 \%$ (Liu et al., 2011). Our patient appeared to have presented in the acute stage with headache, low-grade fever, generalized weakness and signs of meningeal irritation. Although altered consciousness, generalized weakness, cranial nerves palsies, and flaccid paralysis are rare in eosinophilic meningitis these were present in this patient. This phase lasted about six weeks in the patient after which the recovery stage began albeit gradually. 
http://dx.doi.org/10.4314/ajid.v7i1.3

Full recovery was observed at follow up. The recovery phase spanned over six weeks. Eosinophilic meningitis should be considered as a close differential of tuberculous meningitis especially when patient does not seem to be improving with treatment.

\section{Acknowledgement}

The current patient and or/parent is gratefully acknowledged. The support of the nursing staff during the management is highly appreciated.

\section{References}

1. Diao Z, Chen X, Yin C, Wang J, Qi H, Ji A. (2009). Angiostrongylus cantonensis: effect of combination therapy with albendazole and dexamethasone on The cytokine gene expression in PBMC from patients with eosinophilic meningitis. Exp Parasitol. 2009; 123:1-5

2. EOSINOPHILIC MENINGITIS. Infectious Disease Epidemiology Section, Office of Public Health, Louisiana Dept of Health \& Hospitals.

3. Lo Re V3rd., Gluckman SJ., (2003). Eosinophilic meningitis. Am J Med. 114: 217-223.

4. Liu J., Gao J., Zhou C., (2011). A Case Report on Eosinophilic Meningitis Caused by Angiostrongylus cantonensis. Int J Med Sci. 8(6):510-513. Available from http://www.medsci.org/v08p0510.htm.

5. Tsai HC., Lai PH., Sy CL., (2011). Encephalitis caused by Angiostrongylus cantonensis after eating raw frogs mixed with wine as a health supplement. Intern Med. 50(7):771-774

6. Prober CG. (2011). Central Nervous System Infections. In: Kliegman RM, Behrman RE, Jenson HB, Stanton BF, editors. Nelson Textbook of Paediatrics, Saunders . 2047. 